\title{
About the Porous Media Flow Through Circular and Elliptical Anisotropic Inhomogeneities
}

\author{
E. J. M. Veling
}

Received: 24 November 2010 / Accepted: 27 August 2011 / Published online: 28 September 2011

(C) The Author(s) 2011. This article is published with open access at Springerlink.com

\begin{abstract}
In this article, we describe horizontal groundwater flow due to a uniform flow at infinity around a cylindrical or elliptical inhomogeneity, where the permeability inside the inhomogeneity is anisotropic and different from the isotropic domain outside the inhomogeneity. The orientation of the uniform flow with respect to the orientation of the ellipse is arbitrary as well as the orientation of the anisotropy inside the ellipse. We derive an expression for the ratio of the flow through the ellipse with respect to the flow in the homogeneous case.
\end{abstract}

Keywords Analytical solution · Groundwater flow · Anisotropy · Cylindrical inhomogeneity $\cdot$ Elliptical inhomogeneity

\section{Introduction}

In this article, we describe horizontal groundwater flow due to a uniform flow at infinity around a cylindrical or elliptical inhomogeneity, where the permeability inside the inhomogeneity is anisotropic and different from the isotropic domain outside the inhomogeneity. We assume that these inhomogeneities extend over the full height of the aquifer. Study of groundwater flow in a system with many such inhomogeneities gives insight into the characteristics of such aquifers with respect to flow and transport behaviour (Phillips et al. 1991, Sect. 3.8, Zimmerman 1996; Dagan and Lessoff 2001; Dagan et al. 2003; Janković et al. 2003a,b; Suribhatla et al. 2004; Mityushev 2009, among others).

Electronic supplementary material The online version of this article (doi:10.1007/s11242-011-9868-9) contains supplementary material, which is available to authorized users.

\section{E. J. M. Veling $(\varangle)$}

Department of Water Management, Faculty of Civil Engineering and Geosciences, Delft University of Technology, P.O. Box 5048, 2600 GA, Delft, The Netherlands e-mail: E.J.M.Veling@TUDelft.nl 
The remarkable phenomenon of the straight lines of the isohypses and the flow paths inside the inhomogeneity is here present too, but of course, for the anisotropic case, these lines are not any longer perpendicular.

Firstly, we derive the permeability tensor inside the anisotropic inhomogeneity. Next, we study the cylindrical case and thereafter, the elliptic case. We derive an expression for the ratio of the total flow through the inhomogeneity with respect to the homogeneous case. Our main results are (33), (34), and (39). The theory is applied for a number of examples.

The results of this article could be applied in fields as electrical conductivity, thermal conductivity, magnetic permeability, and anti-plane shear displacement in elasticity (with perfectly bonded inclusion), among others, as well.

In principle, this article is self-contained. Details of a number of calculations have been put in Appendices as a separate ESM (Electronic Supplementary Material) file, where it is also shown that the results for the cylinder can be recovered by a suitable limit process applied to the formulas for the elliptic case.

\section{Mathematical Model}

A derivation of the equations steering groundwater flow for a general permeability matrix we refer to Carslaw and Jaeger (1959, pp. 38-49), Bear (1972, pp. 136-147), and Batu (2006, pp. 237-250).

Here, we study the groundwater head of a uniform flow at infinity due to a cylindrical or an elliptical inhomogeneity. The problem for a cylinder is part of the standard hydrology curriculum. The problem for an isotropic ellipsoidal inhomogeneity has been solved before, see Carslaw and Jaeger (1959, pp. 427-428), and for an isotropic elliptical inhomogeneity in different ways by Obdam and Veling (1987) and Dagan and Lessoff (2001). Here, we assume that the permeability inside the inhomogeneity is not isotropic, but anisotropic, where the principal direction $s_{1}$ of the permeability $\left(K_{1}\left[\mathrm{LT}^{-1}\right]\right)$ makes an angle $\phi[-]$ with the positive $X$ axis. The other direction $s_{2}$ is perpendicular to $s_{1}$ and has a permeability of $K_{2}\left[\mathrm{LT}^{-1}\right]$. We assume $K_{1} \geq K_{2}$. Dagan (1989, pp. 195-197) gives the solution for the flow past an isotropic spheroid submerged in an anisotropic matrix, with flow parallel to one of the axes.

The groundwater head $h^{o}[\mathrm{~L}]$ at infinity (and so outside the inhomogeneity) has the representation

$$
h^{o}(x, y)=\widetilde{Q}\left(x \cos \psi^{o}+y \sin \psi^{o}\right), \quad|x|,|y| \rightarrow \infty, \quad \widetilde{Q} \equiv-\left(\frac{Q_{0}}{K^{o} H}\right),
$$

with $K^{o}\left[\mathrm{LT}^{-1}\right]$ the permeability outside the cylinder, $H[\mathrm{~L}]$ the thickness of the aquifer, $Q_{0}\left[\mathrm{~L}^{2} \mathrm{~T}^{-1}\right]$ the discharge per line segment perpendicular to the flow and $\psi^{o}[-]$ the angle with the positive $X$-axis of the natural groundwater flow at infinity and $\widetilde{Q}[-]$ a dimensionless constant. Since the groundwater head is independent of the height in the aquifer, we suppress in the sequel all dependencies in the $z$ direction.

If we denote $q_{\mathrm{s}}\left[\mathrm{LT}^{-1}\right]$ as the specific discharge in the $s$ direction, then the specific discharges outside the inhomogeneity and the Laplace equation hold in the standard way as

$$
\begin{aligned}
& q_{x}^{o}=-K^{o} \frac{\partial h^{o}}{\partial x}, \quad q_{y}^{o}=-K^{o} \frac{\partial h^{o}}{\partial y}, \\
& K^{o}\left(\frac{\partial^{2} h^{o}}{\partial x^{2}}+\frac{\partial^{2} h^{o}}{\partial y^{2}}\right)=0 .
\end{aligned}
$$


In Appendix A (ESM), we derive specific discharges $q_{x}^{i}$ and $q_{y}^{i}$ in terms of $K_{1}, K_{2}$, and $\phi$, with the result

$$
q_{x}^{i}=-K_{x x} \frac{\partial h^{i}}{\partial x}-K_{x y} \frac{\partial h^{i}}{\partial y}, \quad q_{y}^{i}=-K_{y x} \frac{\partial h^{i}}{\partial x}-K_{y y} \frac{\partial h^{i}}{\partial y},
$$

where

$$
\begin{aligned}
& K_{x x}=K_{1} \cos ^{2} \phi+K_{2} \sin ^{2} \phi=\frac{1}{2}\left[\left(K_{1}+K_{2}\right)+\left(K_{1}-K_{2}\right) \cos (2 \phi)\right], \\
& K_{y y}=K_{1} \sin ^{2} \phi+K_{2} \cos ^{2} \phi=\frac{1}{2}\left[\left(K_{1}+K_{2}\right)-\left(K_{1}-K_{2}\right) \cos (2 \phi)\right], \\
& K_{x y}=K_{y x}=\left(K_{1}-K_{2}\right) \sin \phi \cos \phi=\frac{1}{2}\left(K_{1}-K_{2}\right) \sin (2 \phi) .
\end{aligned}
$$

And for convenience, we introduce the shorthand notation $S=-K_{x x}, T=-K_{y y}$, and $U=-K_{x y}$. Inside the inhomogeneity the relation between $q_{x}$ and $q_{y}$ reads

$$
\begin{aligned}
0 & =\frac{\partial q_{x}^{i}}{\partial x}+\frac{\partial q_{y}^{i}}{\partial y}=S \frac{\partial^{2} h^{i}}{\partial x^{2}}+2 U \frac{\partial^{2} h^{i}}{\partial x \partial y}+T \frac{\partial^{2} h^{i}}{\partial y^{2}} \\
& =-\left(K_{x x} \frac{\partial^{2} h^{i}}{\partial x^{2}}+2 K_{x y} \frac{\partial^{2} h^{i}}{\partial x \partial y}+K_{y y} \frac{\partial^{2} h^{i}}{\partial y^{2}}\right),
\end{aligned}
$$

which has to be compared with (3). See Appendix A (ESM) for the relation between $K_{x x}, K_{y y}, K_{x y}$ and $K_{1}, K_{2}, \phi$.

The full mathematical model consists now of the combination of the Eqs. 1, 3, and 6 together with conditions of continuity of the head and the flux at the boundary of the inhomogeneity $\Omega$ :

$$
\begin{array}{ll}
h^{i}(x, y)=h^{o}(x, y), & (x, y) \in \partial \Omega, \\
q_{n}^{i}(x, y)=q_{n}^{o}(x, y), & (x, y) \in \partial \Omega,
\end{array}
$$

where $n$ is the normal direction to the boundary of $\Omega$.

\section{Circular Inhomogeneity}

In this case, the inhomogeneity $\Omega$ is represented as

$$
\Omega=\left\{(x, y, z) \quad \mid x^{2}+y^{2} \leq R^{2}, \quad 0 \leq z \leq H\right\},
$$

with $R[\mathrm{~L}]$ the radius of the cylinder. It is appropriate to use cylindrical coordinates for this problem, so

$$
x=r \cos \theta, \quad y=r \sin \theta,
$$

where $r>0$, and $0 \leq \theta<2 \pi$.

For the translation of the specific discharges $q_{r}^{o}, q_{r}^{i}$ in terms of $K^{o}, K_{1}$, and $K_{2}$ and the head $h^{i}$ and $h^{o}$, we apply the Law of Darcy. Since the outer domain is isotropic there holds

$$
q_{r}^{o}(r, \theta)=-K^{o} \frac{\partial h^{o}}{\partial r}
$$


and that means that at the boundary $\partial \Omega$

$$
q_{r}^{o}(R, \theta)=-K^{o} \frac{\partial h^{o}}{\partial r}, \quad(x=R \cos \theta, \quad y=R \sin \theta) .
$$

The conditions at the boundary $\partial \Omega$ of $\Omega$ can be written as

$$
\begin{aligned}
& h^{i}(x, y)=h^{o}(x, y), \quad(x=R \cos \theta, \quad y=R \sin \theta), \\
& q_{r}^{i}(x, y)=q_{r}^{o}(x, y), \quad(x=R \cos \theta, \quad y=R \sin \theta) .
\end{aligned}
$$

In Appendix B (ESM), we derive expressions for $q_{r}^{i}$ (and $q_{\theta}^{i}$ ). The set of the Eqs. 1, 3, 6, 13, and 14 determine the problem uniquely.

Based on experience in Obdam and Veling (1987), we postulate as solution

$$
h^{i}(x, y)=A x+B y, \quad \text { or equivalently, } \quad h^{i}(r, \theta)=A r \cos \theta+B r \sin \theta,
$$

and

$$
\begin{aligned}
& h^{o}(x, y)=\widetilde{Q}\left(x \cos \psi^{o}+y \sin \psi^{o}\right)+R^{2} \frac{C x+D y}{x^{2}+y^{2}}, \quad \widetilde{Q}=-\left(\frac{Q_{0}}{K^{o} H}\right), \\
& \text { or equivalently, } \\
& h^{o}(r, \theta)=\widetilde{Q}\left(r \cos \theta \cos \psi^{o}+r \sin \theta \sin \psi^{o}\right)+R^{2} \frac{C \cos \theta+D \sin \theta}{r},
\end{aligned}
$$

where $A, B, C$, and $D[-]$ are arbitrary constants for the moment. The requirements (13) and (14) will determine these constants. It can easily be seen that (15), (16) satisfy (6), (3), respectively. At infinity (i.e., $|x|,|y| \rightarrow \infty) h^{o}(r, \theta)$ (16) satisfies (1).

In Appendix C (ESM), we solve for the constants $A, B, C$, and $D$. Finally, we present the solution in the original coordinates $(x, y)$

$$
\begin{aligned}
h^{i}(x, y)=\widetilde{Q}\left(2 K^{o}\right) & {\left[\left\{\left(\frac{\cos ^{2} \phi}{K^{o}+K_{1}}+\frac{\sin ^{2} \phi}{K^{o}+K_{2}}\right) \cos \psi^{o}\right.\right.} \\
+ & \left.\left(\frac{\sin \phi \cos \phi}{K^{o}+K_{1}}-\frac{\sin \phi \cos \phi}{K^{o}+K_{2}}\right) \sin \psi^{o}\right\} x \\
+ & \left\{\left(\frac{\sin \phi \cos \phi}{K^{o}+K_{1}}-\frac{\sin \phi \cos \phi}{K^{o}+K_{2}}\right) \cos \psi^{o}\right. \\
+ & \left.\left.\left(\frac{\sin ^{2} \phi}{K^{o}+K_{1}}+\frac{\cos ^{2} \phi}{K^{o}+K_{2}}\right) \sin \psi^{o}\right\} y\right] .
\end{aligned}
$$

and

$$
\begin{aligned}
h^{o}(x, y)=\widetilde{Q}\left(x \cos \psi^{o}+y \sin \psi^{o}\right) \\
+\widetilde{Q} R^{2}\left[\left[\left\{\frac{\left(K^{o}-K_{1}\right) \cos ^{2} \phi}{K^{o}+K_{1}}+\frac{\left(K^{o}-K_{2}\right) \sin ^{2} \phi}{K^{o}+K_{2}}\right\} \cos \psi^{o}\right.\right. \\
\left.+\left(2 K^{o}\right)\left\{\frac{\sin \phi \cos \phi}{K^{o}+K_{1}}-\frac{\sin \phi \cos \phi}{K^{o}+K_{2}}\right\} \sin \psi^{o}\right] \frac{x}{x^{2}+y^{2}} \\
+\left[\left(2 K^{o}\right)\left\{\frac{\sin \phi \cos \phi}{K^{o}+K_{1}}-\frac{\sin \phi \cos \phi}{K^{o}+K_{2}}\right\} \cos \psi^{o}\right. \\
\left.\left.+\left\{\frac{\left(K^{o}-K_{1}\right) \sin ^{2} \phi}{K^{o}+K_{1}}+\frac{\left(K^{o}-K_{2}\right) \cos ^{2} \phi}{K^{o}+K_{2}}\right\} \sin \psi^{o}\right] \frac{y}{x^{2}+y^{2}}\right] .
\end{aligned}
$$


The inclination $\chi$ of the parallel isohypses inside the cylinder follows from (17) as

$$
\begin{aligned}
\tan \chi & =-\frac{A}{B}=-\frac{\left(T-K^{o}\right) \cos \psi^{o}-U \sin \psi^{o}}{-U \cos \psi^{o}+\left(S-K^{o}\right) \sin \psi^{o}} \\
& =\frac{\left(K^{o}+K_{2} \cos ^{2} \phi+K_{1} \sin ^{2} \phi\right) \cos \psi^{o}+\left(\left(K_{1}-K_{2}\right) \sin \phi \cos \phi\right) \sin \psi^{o}}{\left(\left(K_{1}-K_{2}\right) \sin \phi \cos \phi\right) \cos \psi^{o}-\left(K^{o}+K_{2} \sin ^{2} \phi+K_{1} \cos ^{2} \phi\right) \sin \psi^{o}} .
\end{aligned}
$$

The uniform direction of the flow inside the cylinder follows from (4)

$$
\begin{aligned}
\tan \psi^{i} & =\frac{q_{y}^{i}}{q_{x}^{i}}=\frac{U \frac{\partial h^{i}}{\partial x}+T \frac{\partial h^{i}}{\partial y}}{S \frac{\partial h^{i}}{\partial x}+U \frac{\partial h^{i}}{\partial y}}=\frac{U A+T B}{S A+U B} \\
& =\frac{-K^{o} U \cos \psi^{o}+\left(K_{1} K_{2}-K^{o} T\right) \sin \psi^{o}}{\left(K_{1} K_{2}-K^{o} S\right) \cos \psi^{o}-K^{o} U \sin \psi^{o}},
\end{aligned}
$$

since ST $-U^{2}=K_{1} K_{2}$, see also ESM (C-8).

For the isotropic case $K_{1}=K_{2}$, we have $S=T=-K_{1}, U=0$, and it follows that $\tan \psi^{i}=B / A$, which means that the flow lines are perpendicular to the isohypses inside the cylinder $\left(\tan \psi^{i} \tan \chi=-1\right)$, as it should be.

\section{Elliptical Inhomogeneity}

In this case, the inhomogeneity can be represented as

$$
\Omega=\left\{(x, y, z) \mid \frac{x^{2}}{a^{2}}+\frac{y^{2}}{b^{2}} \leq 1, \quad 0 \leq z \leq H\right\},
$$

where $a, b[\mathrm{~L}]$ are the semimajor and semiminor axes of the ellipse with $a>b$. It is appropriate to use elliptical coordinates for this problem, so

$$
x=c \cosh \xi \cos \eta, \quad y=c \sinh \xi \sin \eta,
$$

where $c^{2}=a^{2}-b^{2}, \xi>0$, and $0 \leq \eta<2 \pi$. The variable $\xi$ measures the size of the ellipse and the variable $\eta$ is the azimuth:

$$
a=c \cosh \xi_{0}, \quad b=c \sinh \xi_{0} .
$$

We introduce also the variable

$$
\tau^{2}=\sinh ^{2} \xi \cos ^{2} \eta+\cosh ^{2} \xi \sin ^{2} \eta=\sinh ^{2} \xi+\sin ^{2} \eta=\cosh ^{2} \xi-\cos ^{2} \eta
$$

In Appendix D (ESM), we derive expressions for the Laplacian and $q_{\xi}^{i}$ (and $q_{\eta}^{i}$ ). Then, conditions at the boundary $\partial \Omega$ of $\Omega$ can be written as

$$
\begin{gathered}
h^{i}\left(\xi_{0}, \eta\right)=h^{o}\left(\xi_{0}, \eta\right), \text { for }\left\{\begin{array}{l}
x=c \cosh \xi_{0} \cos \eta=a \cos \eta, \\
y=c \sinh \xi_{0} \sin \eta=b \sin \eta,
\end{array}\right. \\
q_{\xi}^{i}\left(\xi_{0}, \eta\right)=q_{\xi}^{o}\left(\xi_{0}, \eta\right), \text { for }\left\{\begin{array}{l}
x=c \cosh \xi_{0} \cos \eta=a \cos \eta, \\
y=c \sinh \xi_{0} \sin \eta=b \sin \eta
\end{array}\right.
\end{gathered}
$$

The set of the Eqs. 3, 6, 25, and 26 determine the problem uniquely. 
Again, based on experience in Obdam and Veling (1987), we postulate as solution

$$
\begin{aligned}
h^{i}(x, y)= & A x+B y, \\
& \text { or equivalently, } \\
h^{i}(\xi, \eta)= & A c \cosh \xi \cos \eta+B c \sinh \xi \sin \eta,
\end{aligned}
$$

and, again with $\widetilde{Q}=-\left(\frac{Q_{0}}{K^{o} H}\right)$

$$
\begin{aligned}
h^{o}(\xi, \eta)= & \widetilde{Q} c\left(\cosh \xi \cos \eta \cos \psi^{o}+\sinh \xi \sin \eta \sin \psi^{o}\right) \\
& +c\left(C \mathrm{e}^{-\xi} \cos \eta+D \mathrm{e}^{-\xi} \sin \eta\right),
\end{aligned}
$$

where $A, B, C$, and $D[-]$ are arbitrary constants for the moment. The requirements (25) and (26) will determine these constants $A, B, C$, and $D$. It can easily be seen that (27) satisfies (6) and that (28) satisfies

$$
-\left(K^{o} \frac{\partial^{2} h^{o}}{\partial x^{2}}+K^{o} \frac{\partial^{2} h^{o}}{\partial y^{2}}\right)=-\frac{K^{o}}{c^{2} \tau^{2}}\left(\frac{\partial^{2} h^{o}}{\partial \xi^{2}}+\frac{\partial^{2} h^{o}}{\partial \eta^{2}}\right)=0,
$$

(see $\operatorname{ESM}(\mathrm{D}-14)$ for the derivation). At infinity (i.e., $\xi \rightarrow \infty) h^{o}(\xi, \eta)(28)$ satisfies (1).

In Appendix E (ESM), we solve for the constants $A, B, C$, and $D$, with the results

$$
\begin{gathered}
A=\mathcal{F}\left[\left(T a-K^{o} b\right) \cos \psi^{o}-U b \sin \psi^{o}\right], \\
B=\mathcal{F}\left[-U a \cos \psi^{o}+\left(S b-K^{o} a\right) \sin \psi^{o}\right], \\
C=\frac{\mathcal{F}}{c^{2}}\left[\left\{\frac{K_{1} K_{2}}{K^{o}} a b-K^{o} b^{2}+\left(T a b-S b^{2}\right)\right\} a \cos \psi^{o}-U(a+b) a b \sin \psi^{o}\right], \\
D=\frac{\mathcal{F}}{c^{2}}\left[-U(a+b) a b \cos \psi^{o}+\left\{\frac{K_{1} K_{2}}{K^{o}} a b-K^{o} a^{2}+\left(S a b-T a^{2}\right)\right\} b \sin \psi^{o}\right],
\end{gathered}
$$

where $S=-K_{x x}, T=-K_{y y}$, and $U=-K_{x y}$ (see (5)), and with the definition for the common factor $\mathcal{F}$

$$
\mathcal{F}=\widetilde{Q} \frac{-K^{o}(a+b)}{\left(K^{o 2}+K_{1} K_{2}\right) a b-K^{o}\left(T a^{2}+S b^{2}\right)} .
$$

The inclination $\chi$ of the parallel isohypses inside the ellipse follows from (27) as

$$
\tan \chi=-\frac{A}{B}=-\frac{\left(T a-K^{o} b\right) \cos \psi^{o}-U b \sin \psi^{o}}{-U a \cos \psi^{o}+\left(S b-K^{o} a\right) \sin \psi^{o}} .
$$

The uniform direction of the flow inside the ellipse follows from (4)

$$
\begin{aligned}
& \tan \psi^{i}=\frac{q_{y}^{i}}{q_{x}^{i}}=\frac{U \frac{\partial h^{i}}{\partial x}+T \frac{\partial h^{i}}{\partial y}}{S \frac{\partial h^{i}}{\partial x}+U \frac{\partial h^{i}}{\partial y}}=\frac{U A+T B}{S A+U B} \\
& =\frac{\left.\left\{-K^{o}\left(\left(-K_{1}+K_{2}\right) \sin \phi \cos \phi\right) b\right\} \cos \psi^{o}+\left\{K_{1} K_{2} b-K^{o}\left(-K_{1} \sin ^{2} \phi-K_{2} \cos ^{2} \phi\right) a\right)\right\} \sin \psi^{o}}{\left.\left\{K_{1} K_{2} a-K^{o}\left(-K_{1} \cos ^{2} \phi-K_{2} \sin ^{2} \phi\right) b\right)\right\} \cos \psi^{o}+\left\{-K^{o}\left(\left(-K_{1}+K_{2}\right) \sin \phi \cos \phi\right) a\right\} \sin \psi^{o}} .
\end{aligned}
$$


We remark that for the isotropic case $K_{1}=K_{2}=K^{i}$,

$$
\begin{gathered}
\tan \chi=-\frac{K^{i} a+K^{o} b}{K^{i} b+K^{o} a} \cot \psi^{o}, \\
\tan \psi^{i}=\frac{K^{i} b+K^{o} a}{K^{i} a+K^{o} b} \tan \psi^{o},
\end{gathered}
$$

which means that the flow lines are perpendicular to the isohypses inside the ellipse $\left(\tan \psi^{i} \tan \chi=-1\right)$, as it should be. The expressions for the isotropic case have been found earlier (Obdam and Veling 1987).

In Appendix F (ESM), we show how the results for the circular inhomogeneity can be found in the limit for $c \rightarrow 0$.

\section{Flow Through the Inhomogeneity}

An interesting application of the results found in the former sections is the possibility to find the flow through the inhomogeneity, and in particular the ratio of the flow with respect to the flow in the homogeneous case $\left(K^{o}=K_{1}=K_{2}\right)$. Since the stream function is now not available because of the anisotropy, in contrast to the method in Obdam and Veling (1987), we have to integrate the flux along the border of the inhomogeneity from the two points $\left(\eta_{1}, \eta_{2}\right)$, where the flow inside is tangent to the border of the ellipse. The flow inside is given by (34), and that direction should be equal to

$$
\frac{\mathrm{d} y / \mathrm{d} \eta}{\mathrm{d} x / \mathrm{d} \eta}=-\frac{\sinh \xi_{0} \cos \eta}{\cosh \xi_{0} \sin \eta}=-\frac{b \cos \eta}{a \sin \eta},
$$

so

$$
\tan \psi^{i}=\frac{U A+T B}{S A+U B}=-\frac{b \cos \eta_{i}}{a \sin \eta_{i}}, \quad i=1,2, \quad \text { with } \eta_{2}=\eta_{1}+\pi .
$$

The flow through the inhomogeneity reads

$$
F=\left|\int_{\eta_{1}}^{\eta_{2}} q_{\xi}^{i}\left(\xi_{0}, \eta\right) \sqrt{\left(\frac{\mathrm{d} x}{\mathrm{~d} \eta}\right)^{2}+\left(\frac{\mathrm{d} y}{\mathrm{~d} \eta}\right)^{2}} \mathrm{~d} \eta\right|=c\left|\int_{\eta_{1}}^{\eta_{2}} \tau\left(\xi_{0}, \eta\right) q_{\xi}^{i}\left(\xi_{0}, \eta\right) \mathrm{d} \eta\right|,
$$

where $\tau$ is defined in (24). The flux has been given by ESM (E-1)

$$
\begin{aligned}
q_{\xi}^{i}\left(\xi_{0}, \eta\right)= & \frac{1}{\tau}\left[A\left(S \sinh \xi_{0} \cos \eta+U \cosh \xi_{0} \sin \eta\right)\right. \\
& \left.+B\left(T \cosh \xi_{0} \sin \eta+U \sinh \xi_{0} \cos \eta\right)\right],
\end{aligned}
$$

so, by (35) and (36)

$$
\begin{aligned}
F=c \mid \int_{\eta_{1}}^{\eta_{2}}\left[A\left(S \sinh \xi_{0} \cos \eta+U \cosh \xi_{0} \sin \eta\right)\right. \\
\\
\left.\quad+B\left(T \cosh \xi_{0} \sin \eta+U \sinh \xi_{0} \cos \eta\right) \mathrm{d} \eta\right] \mid \\
=2\left|b(S A+U B) \sin \eta_{2}-a(U A+T B) \cos \eta_{2}\right| .
\end{aligned}
$$


Since, again by (35)

$$
\tan \eta_{2}=-\frac{b(S A+U B)}{a(U A+T B)}, \quad \sin \eta_{2}=\frac{\tan \eta_{2}}{\sqrt{1+\tan ^{2} \eta_{2}}}, \quad \cos \eta_{2}=\frac{1}{\sqrt{1+\tan ^{2} \eta_{2}}},
$$

we have

$$
F=2 \sqrt{a^{2}(U A+T B)^{2}+b^{2}(S A+U B)^{2}} .
$$

For the homogeneous case $\left(K^{o}=K_{1}=K_{2}=K\right)$, we have $S=T=-K^{o}, U=0$, $A=\widetilde{Q} \cos \psi^{o}, B=\widetilde{Q} \sin \psi^{o}$, so

$$
F_{\text {hom }}=2|\widetilde{Q}| K^{o} \sqrt{a^{2} \sin ^{2} \psi^{o}+b^{2} \cos ^{2} \psi^{o}} .
$$

That means that the ratio reads

$$
\mathcal{R}\left(K^{o}, K_{1}, K_{2}, \phi, a, b, \psi^{o}\right)=\frac{F}{F_{\mathrm{hom}}}=\frac{\sqrt{a^{2}(U A+T B)^{2}+b^{2}(S A+U B)^{2}}}{|\widetilde{Q}| K^{o} \sqrt{a^{2} \sin ^{2} \psi^{o}+b^{2} \cos ^{2} \psi^{o}}} .
$$

Some particular cases are

$$
\begin{aligned}
\mathcal{R}\left(K^{o}, K_{1}, K_{2}, 0, a, b, 0\right)= & \frac{K_{1}(a+b)}{K^{o} a+K_{1} b}, \\
\mathcal{R}\left(K^{o}, K_{1}, K_{2}, 0, a, b, \pi / 2\right)= & \frac{K_{2}(a+b)}{K^{o} b+K_{2} a}, \\
\mathcal{R}\left(K^{o}, K_{1}, K_{2}, \pi / 2, a, b, 0\right)= & \frac{K_{2}(a+b)}{K^{o} a+K_{2} b}, \\
\mathcal{R}\left(K^{o}, K_{1}, K_{2}, \pi / 2, a, b, \pi / 2\right)= & \frac{K_{1}(a+b)}{K^{o} b+K_{1} a}, \\
\mathcal{R}\left(K^{o}, K_{1}, 0, \phi, a, b, \phi\right)= & \frac{K_{1} a b+K_{1}\left(a^{2} \sin ^{2} \phi+b^{2} \cos ^{2} \phi\right)}{K^{o} a b+K_{1}\left(a^{2} \sin ^{2} \phi+b^{2} \cos ^{2} \phi\right)}, \\
\mathcal{R}\left(K^{o}, K_{1}, 0, \phi, a, b, \phi+\pi / 2\right)= & \frac{K_{1}\left(a^{2}-b^{2}\right) \sin ^{o} \cos ^{\circ} \phi}{K^{o} a+K_{1}\left(a^{2} \sin ^{2} \phi+b^{2} \cos ^{2} \phi\right)} \\
& \times \sqrt{\frac{a^{2} \sin ^{2} \phi+b^{2} \cos ^{2} \phi}{a^{2} \cos ^{2} \phi+b^{2} \sin ^{2} \phi},}
\end{aligned}
$$

and the corresponding limit cases for a very long, flat ellipse, almost a long slit,

$$
\begin{aligned}
& \lim _{a / b \rightarrow \infty} \mathcal{R}\left(K^{o}, K_{1}, K_{2}, 0, a, b, 0\right)=\frac{K_{1}}{K^{o}}, \\
& \lim _{a / b \rightarrow \infty} \mathcal{R}\left(K^{o}, K_{1}, K_{2}, 0, a, b, \pi / 2\right)= \begin{cases}1, & \text { if } K_{2} \neq 0, \\
0, & \text { if } K_{2}=0,\end{cases} \\
& \lim _{a / b \rightarrow \infty} \mathcal{R}\left(K^{o}, K_{1}, K_{2}, \pi / 2, a, b, 0\right)=\frac{K_{2}}{K^{o}}, \\
& \lim _{a / b \rightarrow \infty} \mathcal{R}\left(K^{o}, K_{1}, K_{2}, \pi / 2, a, b, \pi / 2\right)=1, \\
& \lim _{a / b \rightarrow \infty} \mathcal{R}\left(K^{o}, K_{1}, 0, \phi, a, b, \phi\right)= \begin{cases}1, & \text { if } \sin \phi \neq 0, \\
\frac{K_{1}}{K^{o}}, & \text { if } \sin \phi=0,\end{cases} \\
& \lim _{a / b \rightarrow \infty} \mathcal{R}\left(K^{o}, K_{1}, 0, \phi, a, b, \phi+\pi / 2\right)= \begin{cases}1, & \text { if } \sin \phi \cos \phi \neq 0, \\
0, & \text { if } \sin \phi \cos \phi=0 .\end{cases}
\end{aligned}
$$


For the isotropic case $\left(K_{1}=K_{2}=K^{i}\right)$, we have $S=T=-K^{i}, U=0$,

$$
\begin{aligned}
& A=\frac{K^{o} \widetilde{Q}(a+b)\left(K^{i} a+K^{o} b\right)}{\left(K^{o} a+K^{i} b\right)\left(K^{i} a+K^{o} b\right)} \cos \psi^{o}, \\
& B=\frac{K^{o} \widetilde{Q}(a+b)\left(K^{o} a+K^{i} b\right)}{\left(K^{o} a+K^{i} b\right)\left(K^{i} a+K^{o} b\right)} \sin \psi^{o},
\end{aligned}
$$

so

$$
\begin{aligned}
& \mathcal{R}\left(K^{o}, K^{i}, K^{i}, \phi, a, b, \psi^{o}\right)= \\
& \frac{K^{i}(a+b)}{\left(K^{o} a+K^{i} b\right)\left(K^{i} a+K^{o} b\right)} \frac{\sqrt{a^{2}\left(K^{o} a+K^{i} b\right)^{2} \sin ^{2} \psi^{o}+b^{2}\left(K^{i} a+K^{o} b\right)^{2} \cos ^{2} \psi^{o}}}{\sqrt{a^{2} \sin ^{2} \psi^{o}+b^{2} \cos ^{2} \psi^{o}}} .
\end{aligned}
$$

Introducing the notation $\theta=K^{i} / K^{o}, g=b / a$, we have found this way the same expression as in Obdam and Veling (1987, Eq. 7). Note that in Obdam and Veling (1987), there is a factor 2 missing for the expression for the flow through the ellipse (two lines above Eq. 7 in that reference).

For a cylinder, we find by taking $a=b=R$ in (39):

$$
\mathcal{R}\left(K^{o}, K_{1}, K_{2}, \phi, R, R, \psi^{o}\right)=\frac{\sqrt{(U A+T B)^{2}+(S A+U B)^{2}}}{|\widetilde{Q}| K^{o}} .
$$

A particular case for general $K_{1}$ and $K_{2}$ is

$$
\mathcal{R}\left(K^{o}, K_{1}, K_{2}, \phi, R, R, \phi\right)=\frac{2 K_{1}}{K^{o}+K_{1}},
$$

which is independent of $K_{2}$. This case represents a cylinder where the principal direction $\phi$ is the same as the flow at infinity $\psi^{o}$. The ratio $\mathcal{R}(54)$ is now the same as for the isotropic case (57) below.

Another case is where the principal direction $\phi$ is perpendicular to $\psi^{o}$

$$
\mathcal{R}\left(K^{o}, K_{1}, K_{2}, \phi, R, R, \phi+\pi / 2\right)=\frac{2 K_{2}}{K^{o}+K_{2}} .
$$

This result is of course independent of $K_{1}$. If $K_{2}=0$ in (55), we have

$$
\mathcal{R}\left(K^{o}, K_{1}, 0, \phi, R, R, \phi+\pi / 2\right)=0,
$$

so, there is now no flow at all through the cylinder.

Now, the isotropic case $\left(K_{1}=K_{2}=K^{i}, S=T=-K^{i}, U=0, A=\widetilde{Q} \frac{2 K^{o}}{K^{o}+K^{i}}\right.$ $\left.\cos \psi^{o}, B=\widetilde{Q} \frac{2 K^{o}}{K^{o}+K^{i}} \sin \psi^{o}\right)$ delivers

$$
\mathcal{R}\left(K^{o}, K^{i}, K^{i}, \phi, R, R, \psi^{o}\right)=\frac{2 K^{i}}{K^{o}+K^{i}},
$$

as has been found before (Obdam and Veling 1987). This result is of course independent of $\phi$. 
The function $h^{o}$ is related to the so-called comprehensive potential $\Omega^{o}(u)$ (see Strack 1989; Obdam and Veling 1987) as (with $u=\xi+i \eta$ )

$$
\begin{aligned}
h^{o}= & \frac{1}{K^{o} H} \operatorname{Re} \Omega^{o}(u) \quad \text { with } \\
\Omega^{o}(u)= & \Phi^{o}(u)+i \Psi^{o}(u)=-Q_{0} c \cosh (u) \mathrm{e}^{-i \psi^{o}}+K^{o} H(C+i D) c \mathrm{e}^{-u} \\
= & -Q_{0}\left\{c\left(\cosh \xi \cos \eta \cos \psi^{o}+\sinh \xi \sin \eta \sin \psi^{o}\right)\right. \\
& \left.\quad-i c\left(\cosh \xi \cos \eta \sin \psi^{o}-\sinh \xi \sin \eta \cos \psi^{o}\right)\right\} \\
& +K^{o} H\left\{C c \mathrm{e}^{-\xi} \cos \eta+D c \mathrm{e}^{-\xi} \sin \eta+i\left(-C c \mathrm{e}^{-\xi} \sin \eta+D c \mathrm{e}^{-\xi} \cos \eta\right)\right\} .
\end{aligned}
$$

For the domain inside the inhomogeneity, the theory using complex variables is not applicable since the Laplace equation does not hold there. We can construct a pseudo comprehensive potential $\Omega^{i}(u)$ in such a way, that for $K_{1}=K_{2}=K^{i}$ the functions $\operatorname{Re} \Omega^{i}(u)$ and $\operatorname{Im} \Omega^{i}(u)$ correspond to $h^{i}$ and the stream function $\Psi^{i}(u)$ as follows

$$
\Omega^{i}(u)=\Phi^{i}(u)+i \Psi^{i}(u)=H\left[K^{o}(A x+B y)+i\{(U A+T B) x-(S A+U B) y\}\right],
$$

with

$$
h^{i}=\frac{1}{K^{o} H} \operatorname{Re} \Omega^{i}(u),
$$

and

$$
\Psi^{i}(u)=H\{(U A+T B) x-(S A+U B) y\} .
$$

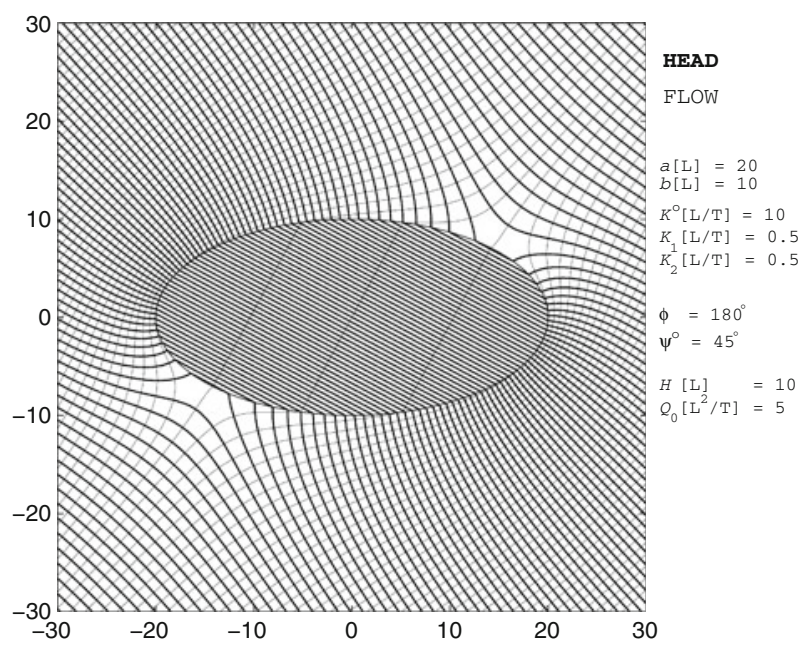

Fig. 1 Example 6.1. Contourlines for the head and stream functions for the isotropic case with $K^{o}=$ $10 \mathrm{~m} / \mathrm{d}, K_{1}=K_{2}=K^{i}=0.5 \mathrm{md}^{-1}$. The head levels run from -2.35 to $2.35 \mathrm{~m}$ relative to some reference head with steps of $0.05 \mathrm{~m}$ and the stream function runs from -189 to $189 \mathrm{~m}^{3} \mathrm{~d}^{-1}$ with steps of $5 \mathrm{~m}^{3} \mathrm{~d}^{-1}$. This figure is identical to Fig. 5 in Obdam and Veling (1987) 


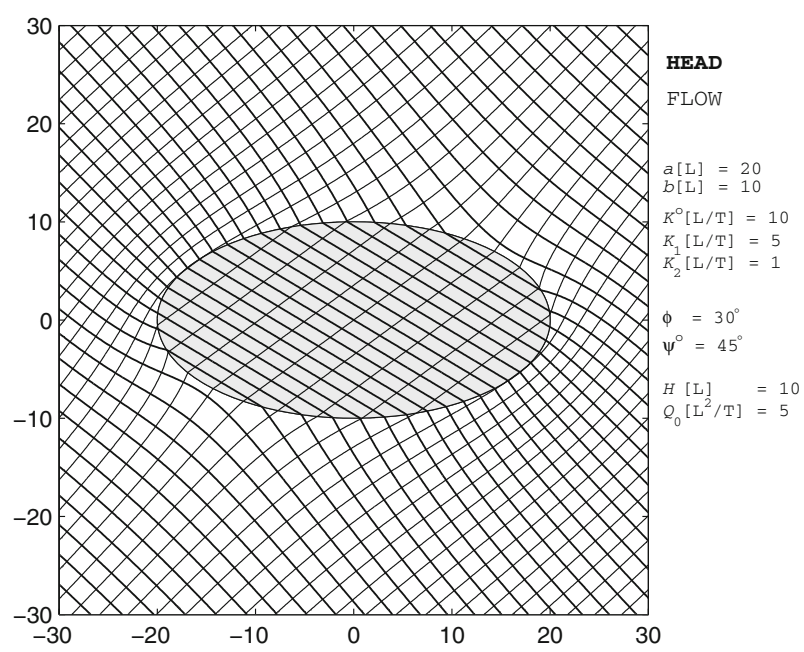

Fig. 2 Case with $\phi=\pi / 6$

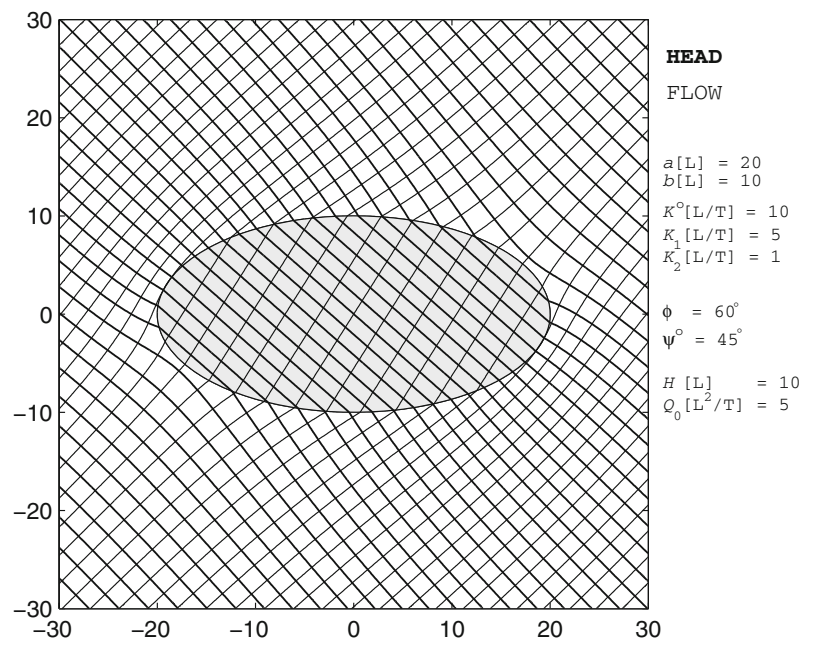

Fig. 3 Example 6.2. Case with $\phi=\pi / 3$

\section{Examples}

Based on the formulas above, we constructed a Matlab ${ }^{\complement}$-script to contour the functions $h^{o}$ and $h^{i}$. Moreover, we use the $\Psi^{o}$ and the $\Psi^{i}$ functions, the imaginary parts of the comprehensive potentials $\Omega^{o}$ and $\Omega^{i}$ (see (58) and (59)):

$$
\begin{aligned}
\Psi^{o}(u) & = \\
\operatorname{Im} \Omega^{o}(u) & =-Q_{0}\left(-x \sin \psi^{o}+y \cos \psi^{o}\right)+K^{o} H\left(-C c \mathrm{e}^{-\xi} \sin \eta+D c \mathrm{e}^{-\xi} \cos \eta\right),
\end{aligned}
$$




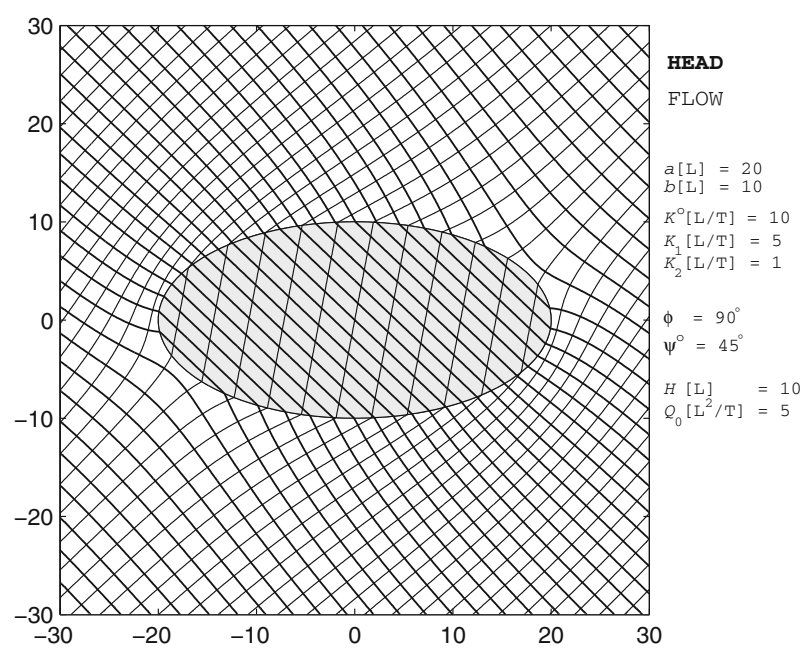

Fig. 4 Example 6.2. Case with $\phi=\pi / 2$

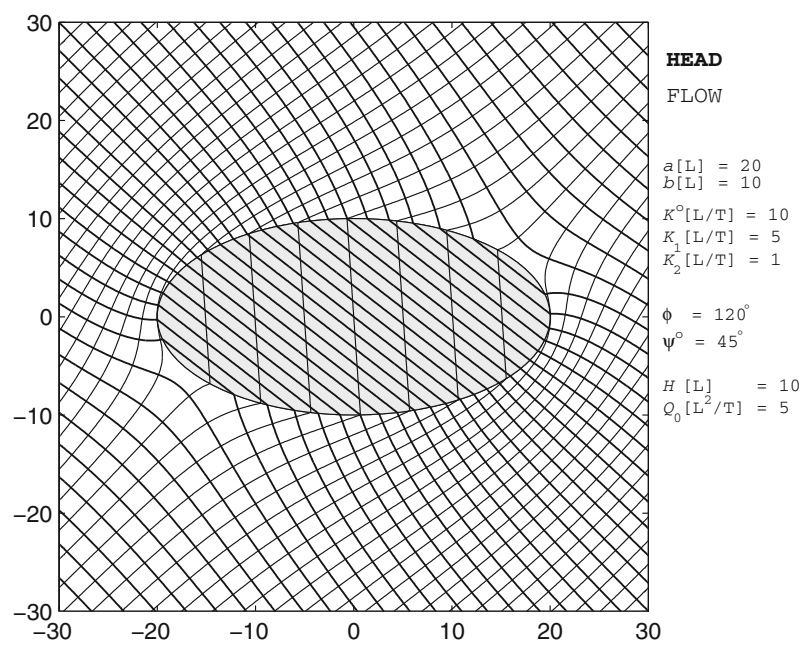

Fig. 5 Example 6.2. Case with $\phi=2 \pi / 3$

$$
\Psi^{i}(u)=
$$

$\operatorname{Im} \Omega^{i}(u)=H K^{i}(A y-B x), \quad$ for the isotropic case, and

$\operatorname{Im} \Omega^{i}(u)=H\{(U A+T B) x-(S A+U B) y\}, \quad$ for the anisotropic case.

Contours of $\Psi^{o}$ and $\Psi^{i}$ give the streamlines.

\subsection{Example 1}

Firstly, we treat the case of an isotropic inhomogeneity as in Obdam and Veling (1987, Fig. 5) and we recover the same isohypses and stream functions, see Fig. 1. 


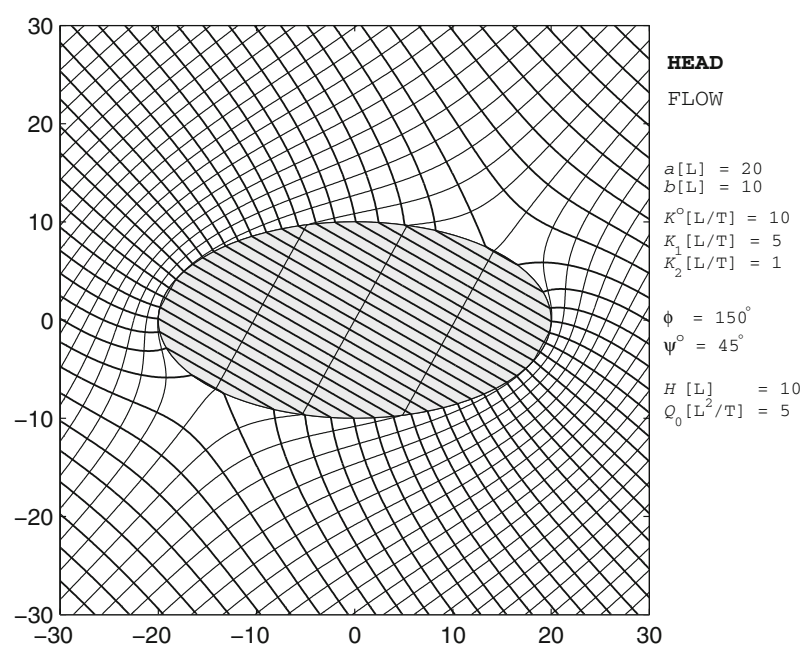

Fig. 6 Example 6.2. Case with $\phi=5 \pi / 6$

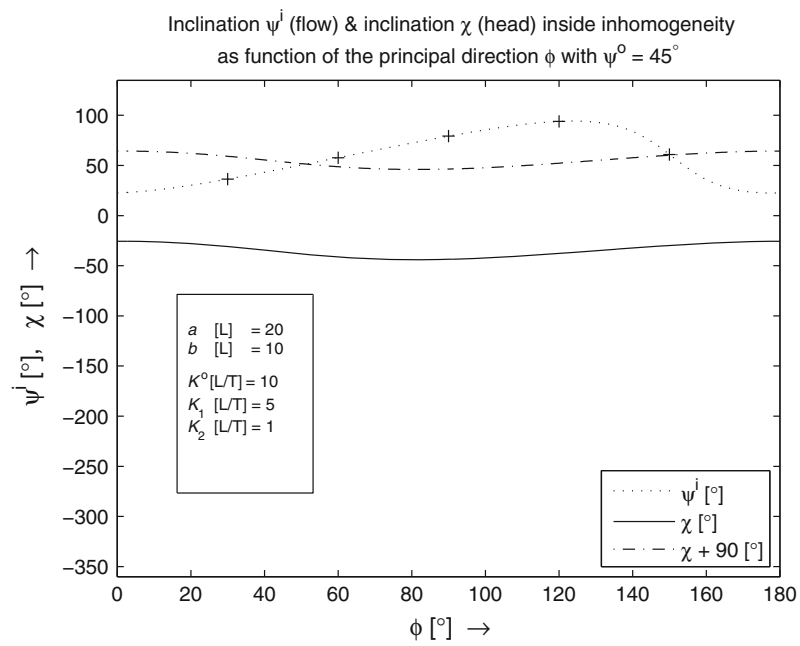

Fig. 7 Example 6.2. Inclination $\psi^{i}$ (flow), $\chi$ (head) and $\chi+90^{\circ}$ inside the inhomogeneity as function of the principal direction $\phi$. Permeability parameters are $K^{o}=10 \mathrm{md}^{-1}, K_{1}=5 \mathrm{md}^{-1}$ and $K_{2}=1 \mathrm{md}^{-1}$ and $\psi^{o}=\pi / 4, a=20 \mathrm{~m}, b=10 \mathrm{~m}$. The ' + ' marks correspond to $\phi=\pi / 6, \pi / 3, \pi / 2,3 \pi / 4$ and $5 \pi / 6$, respectively. As can be seen in Fig. 6 , the inclination for $\phi=5 \pi / 6=150^{\circ}$ has a lower value than that for $\phi=2 \pi / 3=120^{\circ}$ in Fig. 5. For $\phi$ close to $5 \pi / 6$ the isolines are perpendicular (see Fig. 6)

\subsection{Example 2}

Here, we study the influence of the angle $\phi$. For a case with $K^{o}=10 \mathrm{md}^{-1}, K_{1}=5 \mathrm{md}^{-1}$ and $K_{2}=1 \mathrm{md}^{-1}, a=20 \mathrm{~m}, b=10 \mathrm{~h}$, we show the isohypses and stream functions in Figs. 2, 3, 4, 5, and 6 for five different cases with $\phi=\pi / 6, \pi / 3, \pi / 2,2 \pi / 3$ and $5 \pi / 6$, respectively, and all with $\psi^{o}=\pi / 4$.

For this case, we show also the function (34) of the inclination $\psi^{i}$ as function of the principal direction $\phi$, see Fig. 7, for those parameter values. This function is periodic with 


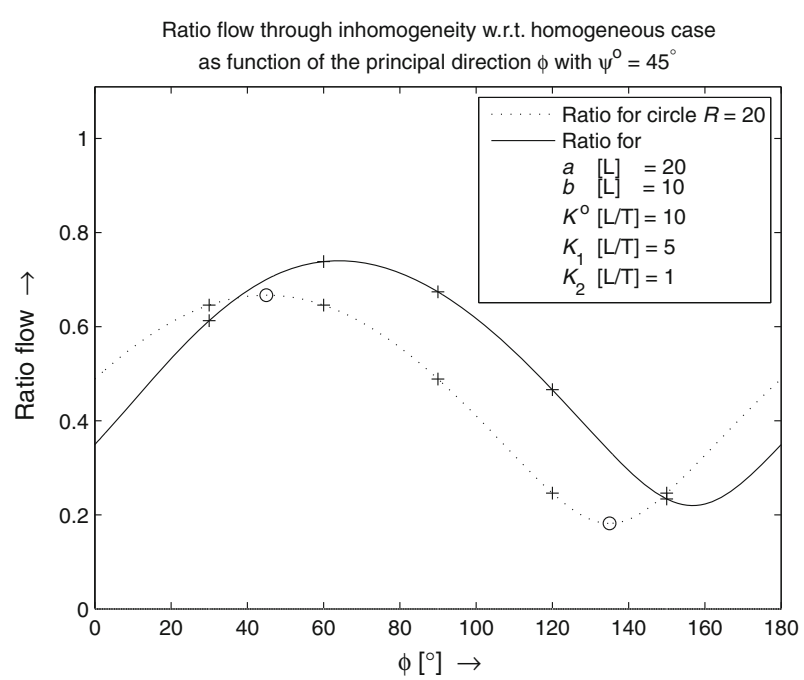

Fig. 8 Example 6.2. Ratio flow through inhomogeneity as function of $\phi$ for an ellipse and circle for $\psi^{\circ}=\pi / 4$. The ratio flow for the circle shows a maximum for $\phi=\pi / 4=45^{\circ}$ and a minimum for $\phi=3 \pi / 4=135^{\circ}$. The ' + ' marks correspond to $\phi=\pi / 6, \pi / 3, \pi / 2,3 \pi / 4$, and $5 \pi / 6$, respectively

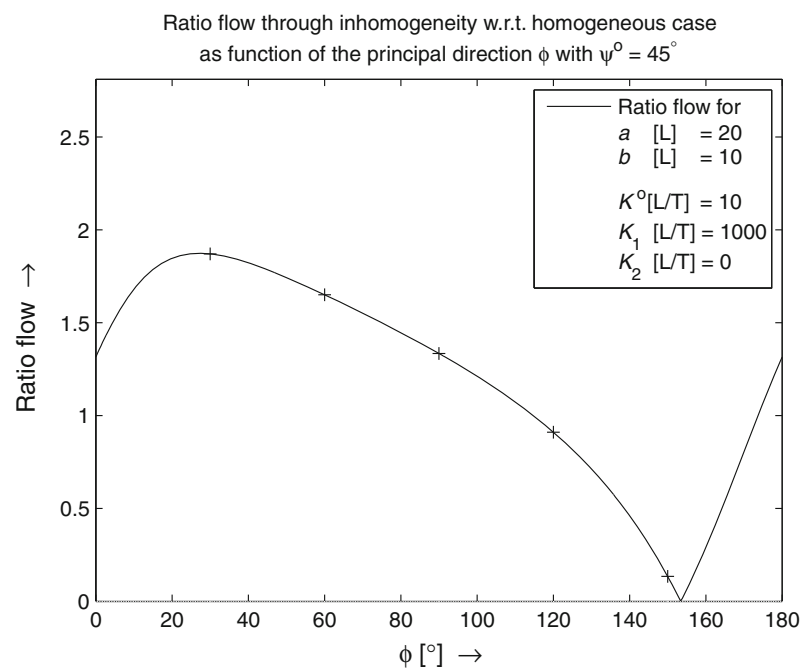

Fig. 9 Example 6.3. Ratio flow through inhomogeneity as function of $\phi$ for an ellipse with a high value for $K_{1}=1000 \mathrm{md}^{-1}$, and $K_{2}=0 \mathrm{md}^{-1}$, and $\psi^{\circ}=\pi / 4$. The ' + ' marks correspond to $\phi=\pi / 6, \pi / 3, \pi / 2,3 \pi / 4$, and $5 \pi / 6$, respectively

period $\pi$, as is clear from the formulas with the terms $\sin ^{2} \phi, \cos ^{2} \phi$ and $\sin \phi \cos \phi$. In the same figure, we show the inclination of the isohypses inside the inhomogeneity. Moreover, the crossings of this inclination added with $90^{\circ}$ with the inclination $\psi^{i}$ give those values for $\phi$ where the iso-lines are perpendicular. As is clear from Fig. 7 this occurs for a value of $\phi$ close to $5 \pi / 6$ as can be seen in Fig. 6. In Fig. 8, the function $\mathcal{R}$ is presented both for this elliptical inhomogeneity and for a cylinder with the same parameters. It is clear that for 


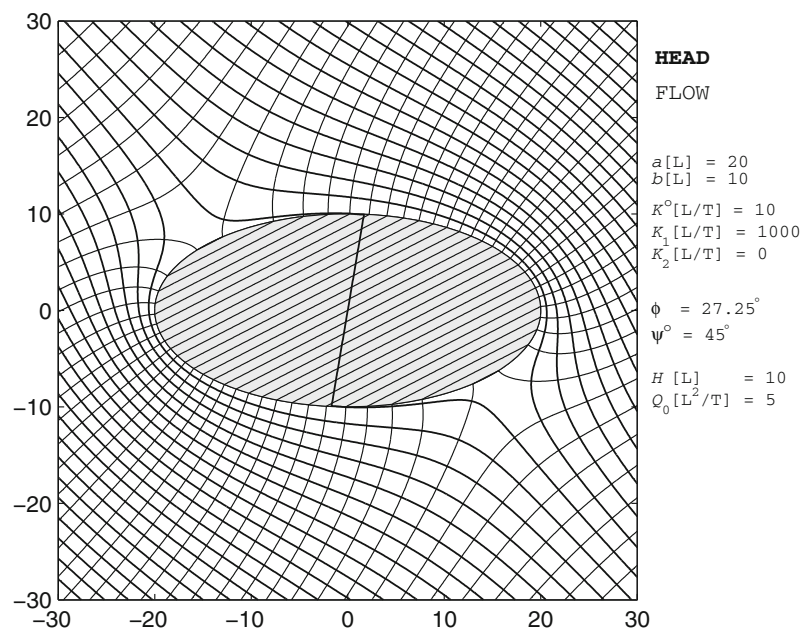

Fig. 10 Example 6.3. Case with high permeability into just one direction $\phi$, so $K_{2}=0 \mathrm{md}^{-1}$. That direction $\phi$ has been chosen such that ratio flow through the inhomogeneity w.r.t. the homogeneous case is maximal, see Fig. 9

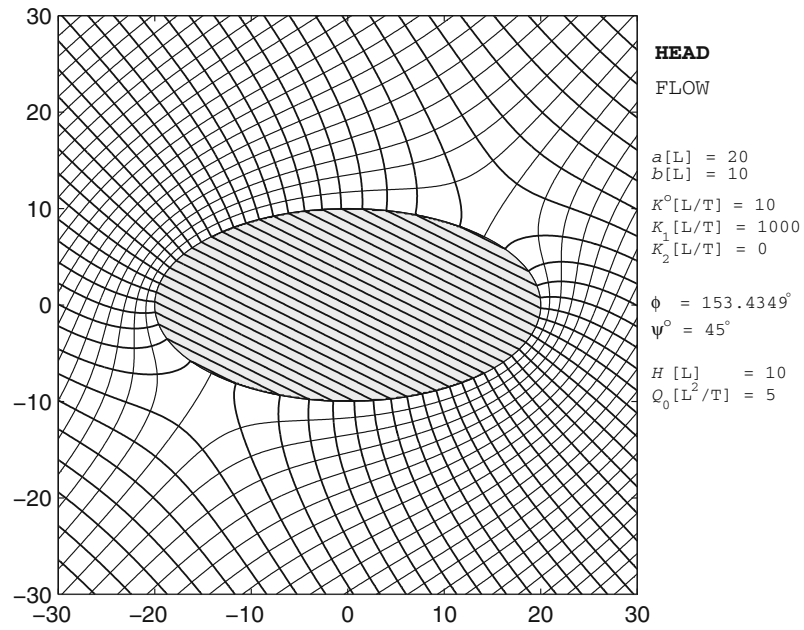

Fig. 11 Example 6.3. Case with high permeability into just one direction $\phi$, so $K_{2}=0 \mathrm{md}^{-1}$. That direction $\phi$ has been chosen such that ratio flow through the inhomogeneity w.r.t. the homogeneous case equals 0 , see Fig. 9. The value for $\phi$ follows from $\tan (\phi)=-b / a$

$\phi=\pi / 4=45^{\circ}$, the flow is maximal for the cylinder, because then $\phi$ has the same value as $\psi^{o}$, while for $\phi=3 \pi / 4=135^{\circ}$ the flow is minimal.

\subsection{Example 3}

Here, we show two cases where inside the inhomogeneity the permeability in one direction is very high and in the direction perpendicular to that direction the ground is impermeable $\left(K^{o}=10 \mathrm{md}^{-1}, K_{1}=1000 \mathrm{md}^{-1}, K_{2}=0 \mathrm{md}^{-1}, \psi^{o}=\pi / 4\right)$. For $K_{2}=0 \mathrm{md}^{-1}$ the 
relation $\psi^{i}=\phi$ always hold, see (34), because of the simplified expressions for $S, T$, and $U$ in that case. In Fig. 9, we show the relative flow through the ellipse as function of $\phi$. In Fig. 10, the direction $\phi=\phi_{\max }$ has been chosen such that the ratio of the flow through the inhomogeneity w.r.t. the homogeneous case is maximal, see Fig. 9. The value for $\chi=\arctan (-A / B)$ changes quickly around $\phi=\phi_{\max }$ since in that neighbourhood the expression for $B$ (which is proportional to $\left.\left(K_{1} \sin \phi \cos \phi\right) a-\left(K_{1} \cos ^{2} \phi\right) b-K^{o} a\right)$ becomes 0 for a value of $\phi_{B}$, with $\phi_{B}$ close to $\phi_{\max }$. We found numerically $\phi_{\max }=27.25001822^{\circ}$, and $\phi_{B}=27.14094131^{\circ}$.

In Fig. 11, the direction $\phi=\phi_{\min }$ has been chosen such that this ratio is 0 . For this example (with $\left.\psi^{o}=\pi / 4\right)$, the value for $\phi_{\min }$ follows from $\tan \left(\phi_{\min }\right)=-b / a=-1 / 2$. That relation holds since for the case $\mathcal{R}\left(K^{o}, K_{1}, K_{2}, \phi, a, b, \psi^{o}\right)=0$ we have both $A U+B T=0$ and $A S+B U=0$, which implies $\tan \chi=-A / B=T / U=\tan \phi$. The last identity holds since $K_{2}=0$, and for $\psi^{o}=\pi / 4$ the expression $-A / B=-b / a$, and so $\tan \phi=-b / a$. We found numerically $\phi_{\min }=153.4349488^{\circ}$.

Acknowledgements The author thanks Christophe Frippiat (Louvain-la-Neuve, Belgium) for suggesting this problem.

Open Access This article is distributed under the terms of the Creative Commons Attribution Noncommercial License which permits any noncommercial use, distribution, and reproduction in any medium, provided the original author(s) and source are credited.

\section{References}

Batu, V.: Applied Flow and Solute Transport Modeling in Aquifers: Fundamental Principles and Analytical and Numerical Methods. Taylor \& Francis, Boca Raton, FL (2006)

Bear, J.: Dynamics of Fluids in Porous Media. American Elsevier, New York (1972)

Carslaw, H.S., Jaeger, J.C.: Conduction of Heat in Solids, 2nd edn. Oxford University Press, Oxford (1959)

Dagan, G.: Flow and Transport in Porous Formations. Springer-Verlag, Berlin (1989)

Dagan, G., Lessoff, S.C.: Solute transport in heterogeneous formations of bimodal conductivity distribution 1. Theory Water Resour. Res. 37(3), 465-472 (2001)

Dagan, G., Fiori, A., Janković, I.: Flow and transport in highly heterogeneous formations: 1. Conceptual framework and validity of first-order approximations. Water Resour. Res. 39(9), 1268 (2003). doi:10. 1029/2002WR001717

Janković, I., Fiori, A., Dagan, G.: Effective conductivity of an isotropic heterogeneous medium of lognormal conductivity distributions. Multiscale Model. Simul. 1(1), 40-56 (2003a)

Janković, I., Fiori, A., Dagan, G.: Flow and transport in highly heterogeneous formations: 3. Numerical simulations and comparison with theoretical results. Water Resour. Res. 39(9), 1270 (2003b). doi:10.1029/ 2002WR001721

Mityushev, V.: Conductivity of a two-dimensional composite containing elliptical inclusions. Proc. R. Soc. A 465(2110), 2991-3010 (2009). doi:10.1098/rspa.2009.0219

Obdam, A.N.M., Veling, E.J.M.: Elliptical inhomogeneities in groundwater flow-an analytical description. J. Hydrol. 95, 87-96 (1987)

Phillips, O.M.: Flow and Reactions in Permeable Rocks. Cambridge University Press, Cambridge (1991)

Strack, O.D.L.: Groundwater Mechanics. Prentice Hall, Englewood Cliffs (1989)

Suribhatla, R., Bakker, M., Bandilla, K., Janković, I.: Steady two-dimensional groundwater flow through many elliptical inhomogeneities. Water Resour. Res. 40, W04202 (2004). doi:10.1029/2003WR002718

Zimmerman, R.: Effective conductivity of a two-dimensional medium containing elliptical inhomogeneities. Proc. R. Soc. Lond. A 452(1950), 1713-1727 (1996). doi:10.1098/rspa.1996.0091 